\title{
2-modem Pursuit-Evasion Problem
}

\author{
Y. Bahoo* \\ A. Mohades ${ }^{\dagger}$ \\ M. Eskandari ${ }^{\ddagger}$ \\ M. Sorouri ${ }^{\S}$
}

\begin{abstract}
In this paper we introduce a new version of the Pursuit-Evasion problem in which the pursuer is a 2 -modem which pursues an unpredictable evader in a polygonal environment. A 2-modem searcher is a wireless device whose radio signal can penetrate two walls. We will present a new cell decomposition of a given polygon $P$ for the 2-modem searcher such that the combinatorial representation of the invisible regions of the searcher remains unchanged. In other words, when the searcher moves inside a cell, no evader can move from an invisible region to another one without detecting by the pursuer.
\end{abstract}

\section{Introduction}

Consider a simple polygon $P$ is given and there are some evaders and a pursuer witch moves continuously in it. The classical pursuit-evasion problem asks for planning the motion of the pursuers in a polygon to eventually see an evader. During the motion of the pursuer, some parts of polygon may be invisible for him; these invisible regions completely depend on the type of the pursuer and its position in $P$. Let $p$ be an arbitrary point in $P$ (as an initial position of the searcher). A maximal connected closed set of points inside $P$ which are invisible for $p$ is called a shadow of $p$. Actually the shadows of $p$ are the subpolygons of $P$ which are denoted by $S_{i}(p)$. As shown in [1], when the searcher moves continuously inside $P$, four geometric events may happen for its shadows: merge, split, appear and disappear. Moreover, when two disjoint shadows of $p$ merge together and make one connected subpolygon, it is called the merge event. In contrast, when a shadow is divided into two components during the motion of the searcher, it is called the split event. Sometimes a shadow is destroyed when the searcher moves; this event is known as the disappear event and if a new shadow is created, we call it the appear event.

In [2], Guibas et al studied the problem of maintaining the distribution of evaders that move out of view

\footnotetext{
* Department of mathematics and computer sciences, Amirkabir university of technology, bahoo@aut.ac.ir

$\dagger$ Department of mathematics and computer sciences, Amirkabir university of technology, mohades@aut.ac.ir

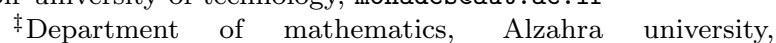
eskandari@alzahra.ac.ir

$\S$ Department of mathematics, Alzahra university, sorouri@alzahra.ac.ir
}

and inferring the location of these targets from combinatorial data extracted by searchers. In this paper, we consider a special type of searchers, 2-modems. As defined in [3], we call a wireless device whose radio signal can penetrate two walls, a 2 -modem. We will present a new cell decomposition of a given polygon $P$ for a 2-modem searcher such that the combinatorial representation of its shadows remains unchanged. In other words, when the searcher moves continuously inside a cell of this decomposition, the merge, appear, disappear or split event does not occur. The study of this problem is motivated by robotics applications such as surveillance, as explained in [2].

\section{The 2-Cell Decomposition}

In this section, we introduce our new decomposition of a given polygon $P$ into convex cells, which provides our main tool for avoiding four events defined above. It is called the 2-cell decomposition.

Definition 1 Let $v$ and $u$ be two vertices of $P$. The vertex $u$ is a critical vertex for $v$ if both of its edges are in the same half-plane bounded by the line $u v$.

The 2-cell decomposition is created by three kinds of lines which are called the partition lines:

1)The lines that are the extensions of both edges of the reflex vertices of $P$ in it;

2) The lines created by a pair of reflex vertices extended towards if both direction are free along the line drawn through the pair of points as shown in [2]; 3)The lines used in the 2-modem visibility polygon of each vertex of the polygon which is introduced in [3].

Now we determine the exact portions of these lines which contribute to the 2-cell decomposition.

\section{Constructing the 2-cell decomposition of $\mathbf{P}$ :}

i) For each reflex vertex of $P$, draw the extensions of its edges until they hit the boundary of $P$.

ii) For each pair of reflex vertices $u$ and $v$ which can see each other $(u v \in P)$, draw the line through $u v$ until it hits the boundary and then omit the segment $u v$. iii) For each vertex $v$ of $P$, construct the 2 -visibility polygon of $v$ as defined in [3].

For illustration of the lines of type 3 which are in the 2 -visibility polygon of the vertices, we provide some examples. Let $u$ be an arbitrary vertex of $P$ and $v$ be a critical vertex of $u$. Figure 1 illustrates the part of the ray $u v$ which is drawn. 


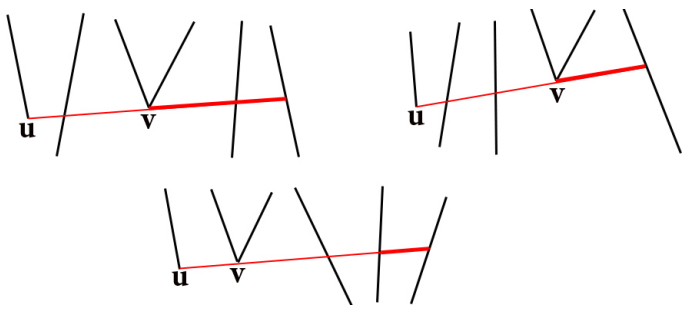

Figure 1: Illustration of constructing the 2-cell decomposition.

Observation 1 The third type of the lines in the 2cell decomposition guaranties neither merge nor split event happens.

Now we would like to show that neither appear nor disappear event occurs when the 2-modem searcher moves inside a cell. For this purpose, we categorize the shadows by having at least one vertex of the polygon inside them or not. Thereby, two types of shadows can be defined as follows:

Definition 2 The type 1 shadow is a shadow which has at least one vertex of the polygon. The type 2 shadow is a shadow which doesn't have any vertices of the polygon; it occurs between two edges of the polygon.

Theorem 1 When the 2- modem searcher moves continuously in a cell, no vertices of the polygon may enter into or exit from its shadow.

Proof. Let $R$ be a cell in the 2-cell decomposition of $P$. At first we will show that if a searcher at an arbitrary point $p$ in $R$ has a shadow of type 1 , during the moving from $p$ inside $R$ no vertices of $P$ can exit from its shadow. Let $q$ be another arbitrary point inside $R$ such that the searcher has moved to $q$. Let $v$ be a vertex of $P$ which is in $S(p)$. Since the cells are convex, the segment $p q$ is completely inside $R$. We suppose for a contradiction that $v$ does not belong to $S(q)$, so the segment $v p$ must intersect the polygon $P$ at least three times, but the segment $v q$ can intersect $P$ at most two times. So there is at least one vertex inside the triangle $p q v$ that is critical for the vertex $v$ (otherwise the vertex $v$ will be visible for the point $p)$. We rotate the ray $\overrightarrow{v q}$ around $v$ towards inside the triangle $p q v$ until reach the first critical vertex for $v$. This vertex is denoted by $r$. The supporting line of the segment $v r$ is one of the partition lines and intersects the segment $p q$. Hence $p$ and $q$ are not in a same cell, a contradiction. Now similarly, we can show that if a searcher (with a shadow of type 1 or type 2 ) moves continuously in a cell, no vertices of $P$ can enter into its shadow.

Notice that by Theorem 1 , we conclude that if a 2modem searcher which has a shadow of type 1 , moves

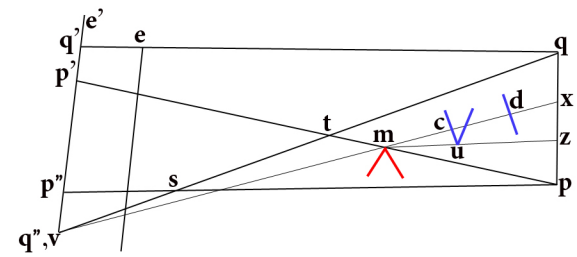

Figure 2: $m x$ intersects $\partial P$ more than once.

in the cell, the appear and the disappear events cannot occur for its shadow. Now we will prove this fact for the case of the type 2 shadow.

Theorem 2 If a 2-modem searcher which is contained in a cell $R$ and has a shadow of type 2 , moves continuously in $R$, the appear and the disappear events cannot occur for its shadow.

Proof. Assume that the 2-modem searcher lies on a point $p$ in $R$ and it has a shadow of type 2 which occurs between two edges of the polygon, named $e$ and $e^{\prime}$. Let $q$ be an arbitrary point inside $R$ such that the searcher moves to $q$. Now we erect a coordinate system with y-axis lined up with the ray $\overrightarrow{p q}$ and the origin at $p$. We connect the point $q$ to the endpoints of $e$ and $e^{\prime}$ and consider two of these line segments for constructing a triangle named $q q^{\prime} q^{\prime \prime}$ such that both segments $q q^{\prime}$ and $q q^{\prime \prime}$ intersect both edges $e$ and $e^{\prime}$, see Figure 2. Also the shadow of the point $p$ occurs between two rays emitted from the point $p$. The intersections of these rays with $e^{\prime}$ are called by $p^{\prime}$ and $p "$. See Figure 2. If there exists a portion of the triangle $q q^{\prime} q "$ enclosed by two edges $e$ and $e^{\prime}$ which is not visible for the point $q$, we are done. Otherwise, suppose that the point $q$ can see the whole of this region. As shown in Figure 2, there is always a vertex of polygon $P$ on the line segment $p p^{\prime}$ such that both edges of $m$ lie below the segment $p p^{\prime}$ (because the shadow of $p$ is started by the ray $\overrightarrow{p p^{\prime}}$ ). The points $s$ and $t$ are the intersections of the line $q q$ " with the lines $p p^{\prime}$ and $p p$ ", respectively. $e_{m}$ is one of the edges of the vertex $m$ which makes the smaller angle with the positive $x$ axis. Now we consider two cases, the supporting line of edge $e_{m}$ intersects the segment $p q$ or not. At first, we suppose that it does not have an intersection with $p q$. In this case, the vertex $m$ should lie on the segment $p t$. For this, we will show that it cannot lie on the segment $t p^{\prime}$. For a contradiction, suppose that $m$ is on the segment $t p^{\prime}$. Since the supporting line of $e_{m}$ does not intersect the segment $p q$, the edge $e_{m}$ will lie below the line $q m$. Therefore a portion of the edge $e^{\prime}$ will be invisible for the point $q$ and this is a contradiction. Consequently, the vertex $m$ should be on the segment $p t$. Now we will show that if $m$ lies on the segment $p t$, the points $p$ and $q$ cannot be in a same cell of our decomposition, which is a contradiction. 
We assume that the vertex $v$ is one of the two vertices of the endpoints of the edges $e$ or $e^{\prime}$ which is on the segment $q q$ ". Since the supporting line of the edge $e_{m}$ does not have an intersection with the segment $p q$ and the vertex $m$ is on the segment $p t$, the line $e_{m}$ should lie below the line $v m$. Therefore the other edge of the vertex $m$ should be below the line $\mathrm{vm}$ (according to the definition of the edge $e_{m}$ ). Hence, the vertex $m$ is a critical vertex for the vertex $v$. We denote the intersection point of the supporting line of $v m$ and the segment $p q$ by $x$ (see Figure 2). Since $p$ and $q$ are inside $P$, the segment $v x$ intersects the polygon, one or more times. If it intersects $P$ at exactly one point, the supporting line of $\mathrm{vm}$ is again one of the partition lines and intersects the segment $p q$ and it means that the points $p$ and $q$ are not in a same cell, a contradiction. So we can assume that the segment $v m$ intersects $P$ in at least two points. First we assume that the segment $v x$ intersects polygon $P$ more than once and the segment $v m$ intersects $P$ just once, see Figure 2 . Note that the segment $m x$ intersects $P$ at least two times (because the number of intersections must be odd). Let $c$ and $d$ denote these intersections. Consider two hypothetical walkers $C$ and $D$ who traverse the boundary of the polygon $P$ such that they enter into the triangle $m x p$, starting from the points $c$ and $d$ respectively. These two walkers must cross the segment $m x$ or the segment $p m$ for leaving triangle $m x p$. Note that since the shadow region $S(p)$ is started by the ray $\overrightarrow{p p^{\prime}}$, at most one of the walkers $D$ or $C$ can cross the segment $p m$, thus at least one of them can cross the segment $m x(C)$. The polygonal chain traversed by the walker $C$ in triangle $m x p$ is denoted by $C H(C)$. We rotate the ray $\overrightarrow{m p}$ around $m$ towards inside the triangle $m x p$ until we reach the first vertex of $C H(C)$. It is clear that it is critical for the vertex $m$. Hence in the triangle $m x p$, there exists at least one vertex of $P$ which is critical for $m$. So we can rotate the ray $\overrightarrow{m p}$ around the point $m$ towards inside the triangle $m x p$, until we reach the first "critical" vertex for $m$ in the triangle $m x p$, which is denoted by $u$. See Figure 2. The intersection of the supporting line of $m u$ and the segment $p q$ is denoted by $z$. We distinguish two cases: the polygon $P$ intersects the segment $m z$ or not. If it intersects the segment $m z$, it will not intersect more than once because the vertex $u$ is the first vertex which is reached by rotating $m x$, so there is a part of the ray us which belongs to the partition lines and intersects the segment $p q$ at the point $z$, a contradiction. Now if the polygon $P$ does not intersect the segment $m z$, so the vertices $u$ and $m$ are reflex and critical for each other, then the segment $u z$ belongs to the partition lines and that is a contradiction. Now we can assume that the segment $v m$ intersects $P$ in at least two points, $a$ and $b$. See Figure 3. Consider two hypothetical walkers $A$ and $B$ who traverse the boundary of the polygon $P$

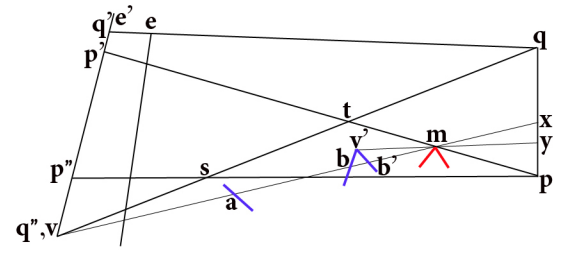

Figure 3: $m v$ intersects $\partial P$ more than once.

such that they enter into the triangle $v m p$, starting from the points $a$ and $b$ respectively. One of these two walkers should pass over the line $v m$. Because otherwise, they should intersect the line $t m$ or the line $v t$. It is clear that both of them cannot leave the triangle $v m p$ from the line $t m$. Because the ray $\overrightarrow{p p^{\prime}}$ determines the border of the shadow region $S(p)$. Thus at least one of them $(A)$ should cross the segment $v t$. In this way, the walker $A$ enters into the triangle $v t p^{\prime}$, and for leaving this triangle it should cross the line $p^{\prime} t$. Because otherwise, it will be an obstacle for the point $q$ (it can only cross the line $q q ")$. On the other hand, the walker $B$ cannot cross the line $t m$, because the walker $A$ intersects the line $p^{\prime} t$. The walker $B$ cannot cross the same line, because the ray $\overrightarrow{p p^{\prime}}$ determines the starting of the shadow region $S(p)$. Also the walker $B$ cannot cross the line $v t$. Because otherwise, it will be an obstacle for $q$ (note that according to our assumption the walker $A$ crosses the line $v t$ ). So the walker $B$ should intersect the line $\mathrm{vm}$. The polygonal chain traversed by the walker $B$ in triangle $v t m$ is denoted by $C H(B)$. We rotate the ray $\overrightarrow{m t}$ around $m$ towards inside the triangle $v t m$ until we reach the first vertex of $C H(B)$. Obviously, this vertex is critical for the vertex $m$. Then we can assume that there exists always a critical vertex for $m$ in the triangle $v t m$. So we can rotate the ray $\overrightarrow{m t}$ around the point $m$ towards inside the triangle $v t m$, until we reach the first "critical" vertex for $m$ in the triangle $v t m$, which is denoted by $v^{\prime}$. It is clear the vertices $v^{\prime}$ and $m$ are critical for each other. The intersection of the ray $\overrightarrow{m v^{\prime}}$ and the segment $p q$ is denoted by $y$. Note that the segment $m v^{\prime}$ intersects $P$ at most once (otherwise the vertex $v^{\prime}$ is not the first critical vertex). We distinguish two cases: the polygon $P$ doesn't intersect the segment $m v^{\prime}$ or just one intersection occurs. In the first case, it is clear that the vertices $v^{\prime}$ and $m$ are reflex and critical for each other, so the supporting line of $v^{\prime} m$ will be one of the partition lines and intersects the segment $p q$ and it means that the points $p$ and $q$ are not in a same cell, a contradiction. In the second case, when the polygon $P$ just has one intersection with the supporting line of $m v^{\prime}$, there exists a part of the segment my which belongs to a partition line. It can be shown that the points $p$ and $q$ are not in a same cell which is a contradiction (similar to the way described above for the case of the vertex $u$ is a critical vertex 
for the vertex $m$ ). According to the above discus-

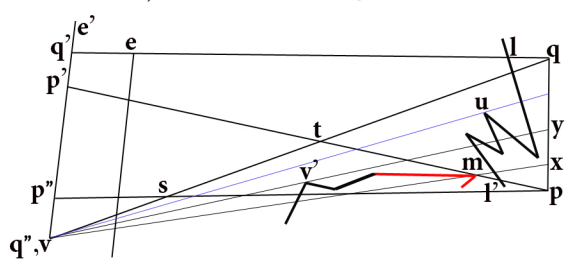

Figure 4: $e_{m}$ intersects $p q$.

sion, we have reached a contradiction when the edge $e_{m}$ does not intersect the segment $p q$, hence the supporting line of $e_{m}$ must intersect the segment $p q$. See Figure 4 . Note that the point $q$ is inside the polygon $P$, then the segment $q q^{\prime}$ must have at least another intersection with the polygon, except at $e$. Also, we know that the point $q^{\prime}$ is visible for $q$, hence the segment $q q^{\prime}$ should intersect $P$ at most once, except at $e$. This intersection is denoted by $l$. Now we consider a hypothetical walker $L$ who traverses the boundary of $P$ inside the triangle $q q^{\prime} q$ ". This polygonal chain is denoted by $C H(L)$. The walker $L$ should intersect the segment $p p^{\prime}$ for leaving the quadrilateral $p^{\prime} q^{\prime} p q$. This intersection point is denoted by $l^{\prime}$. We distinguish two cases: the point $l^{\prime}$ lies on the segment $m p^{\prime}$ or $p m$. In both cases we will reach a contradiction. First, we suppose the point $l^{\prime}$ lies on the segment $m p^{\prime}$. In this case, it is clear that the segment $m q$ cannot intersect the polygon $P$ (otherwise the point $q^{\prime}$ is invisible for the point $q$ or $P$ is not a simple polygon). In addition, it is easy to show that the vertex $m$ is a reflex vertex (because of the number of intersections between the segment $m q$ and $P$ and $q$ is an interior point of $P$ ). Thus the supporting line of $e_{m}$ is one of the partition lines and intersects the segment $p q$ and it means that the points $p$ and $q$ are not in a same cell, a contradiction. In the second case where we consider the point $l^{\prime}$ lies on the segment $m p$, the vertex $m$ must be on the segment $t p$. Otherwise, since $l^{\prime}$ is on the segment $m p$, the polygonal chain traversed by walker $L$ from $l$ to $l^{\prime}$ is intersected by the segment $m q$, so $q$ cannot see whole segment $q q^{\prime}$. Therefore the edge $e_{m}$ will be inside the triangle $v t m$ and it cannot cross the segment $t m$ (note that the shadow region $S(p)$ is started by the ray $\left.\overrightarrow{p p^{\prime}}\right)$, furthermore it cannot cross the segment $t v$ (because $L$ crosses the segment $q q$ " once, hence the vertex $q$ " is not visible by $q$ ). Thus the polygonal chain in the triangle $v t m$ crosses the segment $v m$ at least one time. Thereby there exists always one vertex inside the triangle tvm. We rotate the ray $\overrightarrow{v t}$ around the point $v$ towards inside the triangle $v t m$, until we reach the first "critical" vertex for $v$ in the triangle $v t m$ (because $v^{\prime}$ is the first vertex, the polygon $P$ doesn't intersect the segment $q q$ " and the vertex $q$ should see $q ")$. This vertex is denoted by $v^{\prime}$. The intersection of the supporting line of $v v^{\prime}$ and the segment $p q$ is denoted by $y$. If the segment $v^{\prime} y$ intersects the polygon just once (on $C H(L)$ ), the supporting line of $v^{\prime} y$ is one of the partition lines (because vertex $v^{\prime}$ is a critical vertex for the vertex $v$ ) and intersects the segment $p q$ and it means that the points $p$ and $q$ are not in a same cell, a contradiction.

Then we suppose that segment $v^{\prime} y$ intersect $P$ more than once. All of these intersections are inside the triangle $t q p$ (when the ray $\overrightarrow{v t}$ is rotated towards inside the triangle $v t m$, the vertex $v^{\prime}$ is the first vertex). The segment $q q$ " should intersect the polygon $P$ just on $C H(L)$ ( $q$ should see $q$ "). So if the segment $v y$ intersects the polygon $P$ except on $C H(L)$, then there exists a vertex inside region $S=\triangle t p q \cap \triangle q q$ " $y$. We rotate the ray $\overrightarrow{v q}$ around the vertex $v$ toward in triangle $t p q$ until we reach the first "critical" vertex for $v$ in the region $S$ which is denoted by $u$. The supporting line of $u v$ intersects $P$ just once on $C H(L)$ (otherwise the vertex $v^{\prime}$ is not the first critical vertex). Thus the supporting line of $u v$ is one of the partition lines and intersects the segment $p q$ and it means that the points $p$ and $q$ are not in a same cell, a contradiction. In all above cases, we showed that there is always a part of the segment $q^{\prime} q$ " which is not visible from $q$. Hence $q$ should have a shadow between $e$ and $e^{\prime}$.

Also in a similar way, it can be shown that no appear event occurs.

Due to Theorems 1 and 2, when a 2-modem searcher moves continuously in a cell, neither disappear nor appear event can happen, hence the 2-cell decomposition guaranties that the combinatorial representation of the invisible regions of the searcher remains unchanged.

\section{Conclusion}

In this study, we considered a new version of PursuitEvasion problem and introduced a new decomposition of a given polygon into convex cells which assures that no evader can move from an invisible region to another one without detecting by the pursuer while the searcher moves inside a cell. Moreover it can be shown that the number of cells in the 2-cell decomposition is $O\left(n^{3}\right)$, but it takes a bit of effort. Also the complexity of the algorithm is the same.

\section{References}

[1] J. Yu and S. M. LaValle. Shadow information spaces: Combinatorial filters for tracking targets. IEEE Transactions on Robotics, 28(2):440-456, 2012.

[2] L. J. Guibas, J.-C. Latombe, S. M. LaValle, D. Lin, and R. Motwani. Visibility-based pursuit-evasion in a polygonal environment. International Journal of Computational Geometry and Applications, 9(5):471-494, 1999

[3] A. M. Martins. Geometric optimization on visibility problems Ph.D. thesis, 2009, Aveiro University. 